\title{
The Influence of Temperature and Ecosystem Dynamics on the Partitioning of a Persistent Organic Pollutant (POP) in Antarctic Marine Food Webs
}

\author{
R.A. Cropp ${ }^{\text {a }}$, G.M. Kerr ${ }^{\text {a }}$, S. M. Bengtson-Nash ${ }^{\text {a }}$ and D.W. Hawker ${ }^{\text {a }}$ \\ ${ }^{a}$ Atmospheric Environment Research Centre, School of Environment, Griffith University, Nathan, Qld. 4111, \\ Australia \\ Email: R.Cropp@griffith.edu.au
}

\begin{abstract}
Persistent organic pollutants (POPs) are toxic man-made chemicals that do not readily degrade in the natural environment, and may be subject to long-range environmental transport, potentially reaching remote regions far from the emission sources. Chlorinated POPs are hydrophobic and lipophilic chemicals with octanol-water partition coefficients $\left(K_{\mathrm{OW}}\right)$ typically of the order of $10^{4}$ to $10^{7}$. This combination of properties means that POPs have a propensity to bioconcentrate and bioaccumulate in living organisms, representing a significant risk to human and environmental health (Wania and Mackay, 1999). The movement of chemicals such as POPs in the environment can be described using the concept of fugacity (Mackay et al., 2009). Fugacity (literally 'fleeing tendency', $f$ ) is an equilibrium criterion related to chemical potential that determines how a chemical will partition between multiple phases (Mackay, 2001). The rate of chemical movement between phases is proportional to the difference in fugacity. A chemical in diffusive equilibrium between two phases is unlikely to have the same concentration in the phases, but it will have the same fugacity.
\end{abstract}

Whilst it is known that high latitude environments can act as repositories for semi-volatile POPs, little is known about the movement and behavior of POPs in Antarctic ecosystems. Polar marine environments are characterised by strong, seasonal variations in light and temperature that affect primary productivity and thereby drive the population dynamics of polar biological systems (De Laender et al., 2010). Spring and summer phytoplankton blooms play a major role in biogeochemical cycling and potentially also in cycling of POPs. Temperature changes also affect the partitioning of POPs in the environment. To provide useful insights into the seasonal behavior and fate of these compounds in Antarctic ecosystems, any model simulation must accommodate the large seasonal plankton biomass changes as well as temperature variation. The approach adopted involved fugacity-based models of the physical and biological environments together with a dynamic plankton food web model. The physical fugacity model and the plankton food web model may be considered sub-models that are coupled by the biological fugacity model (Cropp et al., 2011).

This work then describes a mass-conserving, fugacity-based model and its application to investigate the effect of seasonal variations in temperature and light on the movement of POPs in Antarctic physical and plankton systems. The use of a plankton ecosystem model to simulate the movement of POPs requires that a detritus compartment, composed of dead phytoplankton and zooplankton and their respiration products, be included. This is because it provides a pathway for POPs in dead organic matter to exchange with the water. The model is applied to the movement of hexachlorobenzene (HCB), still a prevalent POP in remote regions (Weber and Goerke, 2003).

The model reveals that seasonal variations in temperature and plankton biomass have different impacts on the partitioning of POPs in polar environments. It predicts that the burden of HCB in the plankton varies with the seasonal cycle of plankton biomass in Antarctic waters, and that this variation is primarily due to plankton dynamics. Seasonal variations in temperature have little effect on the distribution of HCB in terms of mass, with the exception of detritus in which it generates a subtle seasonal cycle. Temperature variations do affect fluxes between the physical compartments of the model (especially diffusive exchange between water and air, and water and sediment), but have negligible effect on the mass distribution of HCB in these compartments.

Keywords: Fugacity, Plankton, Detritus, Dynamic coupled food web model 


\section{INTRODUCTION}

POPs such as HCB are generally semi-volatile compounds. Volatilised fractions of legacy POPs and molecules adsorbed to fine particles may be transported great distances by air masses where they will undergo global fractionation processes along temperature gradients, favouring deposition in colder climates such as high altitude or latitude environments. The polar regions of the earth have been proposed as significant environmental repositories for many chemicals that can be transported by natural processes from their areas of use, and this view has been supported by direct observations of chemical distribution trends, primarily from the Arctic region (e.g. Kelly et al., 2008). By contrast, the Antarctic region has not received the same degree of scrutiny (Bengtson-Nash, 2011). Antarctic plankton ecosystems are generally simpler than their Arctic counterparts (Corsolini et al., 2002). The dominant grazer species in Antarctic plankton ecosystems is the zooplankton species Euphausia superba (Antarctic krill), which forms the base diet of the majority of marine mammal predators (Bengtson-Nash et al., 2008). Krill feed on phytoplankton, of which one of the most abundant species in Antarctic waters is the prymnesiophyte Phaeocystis spp.

Polar marine environments are characterised by strong, seasonal variations in light and temperature that affect primary productivity and thereby drive the population dynamics of polar biological systems (De Laender et al., 2010). The influence of the dynamics of the biological system on the movement of POPs within the physical system, particularly in environments such as Antarctic waters, is not well understood. The large plankton blooms typical of the Antarctic seasonal cycle produce large quantities of lipids, into which POPs partition in preference to other environmental phases or compartments such as air and water drive, the population dynamics of polar biological systems (Dachs et al., 1999).

The nutrient-phytoplankton-zooplankton (NPZ) model is a generic tool used to describe the dynamics of many plankton ecosystems and is the optimal approach to modelling plankton ecosystems in the absence of detailed measurements of the population dynamics, as is the case in Antarctic marine plankton systems. NPZ models are mechanistic process-based models that include representations of the phytoplankton uptake of nutrients, zooplankton consumption of phytoplankton and implicit microbial recycling of biological waste products back into the dissolved inorganic nutrient pool. They are often written in a currency of the limiting inorganic nutrient, which in the ocean is commonly nitrogen (Franks, 2002). In applying an NPZ model to modeling the movement of POPs through an ecosystem, a detritus compartment ( $D$, composed of dead phytoplankton and zooplankton and their respiration products) should be included, resulting in an NPZD model. Detritus provides the opportunity for POPs in dead organic matter to diffusively exchange with the water.

The movement of chemicals such as POPs in the environment can be described using the concept of fugacity (Mackay et al., 2009). Fugacity is related to chemical potential and determines how a chemical will partition between multiple phases (Mackay, 2001). The rate of chemical movement between phases is proportional to the difference in fugacity. The fugacity $\left(f_{i}\right)$ of a phase $i$ is measured as a pressure (e.g. Pa) and is related to concentration in the phase $\left(C_{i}\right.$, e.g. $\left.\mathrm{mol} \mathrm{m}^{-3}\right)$ by the expression:

$$
C_{i}=Z_{i} f_{i}
$$

where $Z_{i}$ is the fugacity capacity constant $\left(\mathrm{mol} \mathrm{m}^{-3} \mathrm{~Pa}^{-1}\right)$ of phase $i$. A fugacity-based model of the physical environment that describes the partitioning of POPs amongst the air, water and sediment compartments, and a fugacity-based model that describes the partitioning of POPs among the compartments of a NPZD model are constructed. The physical fugacity model and the plankton food web model may be considered submodels that are coupled by the biological fugacity model (Cropp et al., 2011). It is recognized that POPs typically move through the environment in association with carbon, and therefore only the lipid content of the biota is coupled to the fugacity model, assuming constant Redfield ratios (Reynolds, 2006). The physical and ecosystem fugacity-based models are relatively common and described in more detail by Cropp et al. (2011).

The dynamic fugacity-based approach to model the movement of POPs in physical and biological systems has not to our knowledge been extensively applied to an Antarctic system. In this work we develop a fully coupled dynamic fugacity-based model that is driven by seasonal changes in the growth rate of phytoplankton that result from changes in the light intensity and sea surface temperature. Despite large seasonal changes in plankton biomass, the total mass of POPs in our closed physical-biological system remains constant over time. An axiomatic principle of models describing the movement of matter in an environment is that mass should be conserved (Wania and Mackay, 1999). 


\section{METHODS}

\subsection{Model Definition, Calibration and Paramerization}

The physical domain of the model was defined as a representative region of the Southern Ocean on the Antarctic continental shelf. It was assumed that the region is permanently free of sea ice. Specifically, it was a $1-\mathrm{m}^{2}$ column of air, water and sediment that begins at the top of the atmospheric marine boundary layer, at a notional height of one kilometre, and extends through a mid-continental shelf water column of 100-m depth. It terminates in a layer of sediment on the ocean floor $0.05-\mathrm{m}$ thick representing that part of the sediment that actively exchanges POPs with the overlying water. The physical domain assumes that the atmospheric and ocean layers are fully mixed and homogeneous, and that the whole of the sediment compartment exchanges POP with the water column above. The initial values for the NPZD model parameters, obtained from values reported in the literature were used as starting values to calibrate the NPZD model to reproduce observed plankton dynamics in the study area. The model was calibrated in a two-stage process. First the forced ecosystem model was calibrated to reproduce an eight-year climatology of surface ocean chlorophyll- $a$ concentrations measured by satellite using the Sea-viewing Wide-Field-of-View Sensor (SeaWiFS, Hooker and McClain, 2000) in an area of the Southern Ocean near the Antarctic coast $\left(60-65^{\circ} \mathrm{S}\right.$,

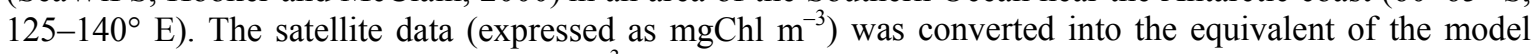
output of phytoplankton biomass $\left(\mathrm{mgN} \mathrm{m}^{-3}\right)$ using Redfield ratios: a nitrogen-to-carbon ratio of 0.175 and a carbon-to-chlorophyll ratio of 50 (Gabric et al., 2001). The calibrated ecosystem parameter values were then used for the calibration of the fugacity sub-models. Measured concentrations of HCB in air, water, sediment, phytoplankton and zooplankton (with the exception of detritus because of a lack of data), reported in recently published literature suggested that a total HCB mass of $5 \times 10^{-7}$ mol was appropriate for the defined model domain.

\subsection{Temperature Correction}

The relevant temperature dependent physico-chemical properties of HCB were corrected to reflect seasonal changes in temperature for the study region using the Van't Hoff expression. This relates a parameter $(X)$ at a specific temperature $(T)$ to a reference value $\left(X_{\mathrm{R}}\right)$ at a reference temperature $\left(T_{\mathrm{R}}\right)$ using the change in enthalpy $\left(\Delta H_{X}\right)$ or change in internal energy temperature

$$
X(T)=X_{\mathrm{R}}\left(T_{\mathrm{R}}\right) e^{\frac{-\Delta U_{X}}{R}\left(\frac{1}{T}-\frac{1}{T_{\mathrm{R}}}\right)}
$$

The Henry's law constant $(H)$, the octanol-water partition coefficient $\left(K_{\mathrm{OW}}\right)$ and the sub-cooled liquid vapour pressure $\left(\underline{P}_{1}^{\mathrm{s}}\right)$ were corrected for temperature using a reference temperature $\left(T_{\mathrm{R}}\right)$ of $298 \mathrm{~K}$ and the internal phase transfer energies of HCB as described by Shen and Wania (2005). These corrected values were used to calculate seasonal climatologies of temperature-dependent fugacity capacity constants. The sea surface temperature (SST) data and monthly mean air temperatures sourced from a thirty year archive measured at the Dumont D'urville base (Stearns et al., 1993) located just south of the study region $\left(66^{\circ} 53^{\prime} \mathrm{S}, 140^{\circ} 30^{\prime} \mathrm{E}\right)$, were used to dynamically correct the fugacity capacities of the compartments of the fugacity sub-model.

The seasonal variation in water temperature is much smaller than, and out of phase with, the seasonal variation in air temperature in the model environment. The difference in air and water phase temperature, and its subsequent effect on air-water movement of POPs was addressed by separating the overall air-water transport parameter $\left(D_{\mathrm{AW}}\right)$ into phase specific values with temperature dependent $Z$ values employed as appropriate (Wania and Mackay, 1995). The temperature data were used with equation (2) to generate a seasonal climatology of the fugacity capacity constant of each compartment. A cubic spline was then numerically fitted to each time series and differentiated to give a time series of fugacity capacity correction terms for each compartment. This allowed us to use an adaptive step size integration routine that permitted enforcement of our conservation of mass criterion to machine epsilon.

\subsection{Dynamic Simulations}

The growth rate of the phytoplankton in the model is sensitive to seasonal changes in the sea surface photosynthetically active radiation (PAR), the depth of the surface ocean mixed layer (MLD) and SST measured in an area of the Southern Ocean near the Antarctic coast $\left(60-65^{\circ} \mathrm{S}, 125-140^{\circ} \mathrm{E}\right)$. The relationships used to model the effect of changes in the light field and temperature on phytoplankton growth rate, are found in Cropp et al. (2011). The seasonally forced model equations were integrated over a multi-year timespan until a repeating annual cycle was obtained. This ensured that the initial conditions did not affect 
the results, as we were interested in a 'typical' annual cycle rather than a response to a particular perturbation.

For the purposes of this work, simulations were also run in which the fugacity capacities of the compartments were not corrected for variations in temperature in order to be able to differentiate POP movements that resulted from ecosystem dynamics from those associated with changes in temperature. The results of these simulations are the basis for our attribution of HCB fluxes to ecosystem processes or temperature variation.

\section{RESULTS}

Seasonal variations in irradiance, mixed layer depth and temperature generate significant annual cycles in plankton populations in Antarctica. The simulated phytoplankton population peaks in early summer, consistent with satellite observations, and although the zooplankton population begins to grow as the phytoplankton population increases, there is a significant temporal lag before the zooplankton population reaches its maximum biomass. Detritus also evidences an annual cycle with a peak lagging by a few days the peak in zooplankton biomass, confirming the zooplankton respiration and excretion as the major sources of detritus in the model.

The seasonal cycle of the plankton biomass induces a seasonal cycle in the fugacity of HCB of each phase in addition to seasonal variations of fugacity of both biological and physical compartments because of the temperature dependence of the fugacity capacities. The concentration of HCB in each compartment scales linearly with fugacity (equation (1)), so the seasonal variations in concentration of HCB in each phase are identical to those of fugacity.

To explore the separate effects of seasonal plankton biomass and temperature changes separately, the model was run with constant temperature and constant ecosystem parameters respectively. Figure 1 shows the HCB fugacity of each compartment, physical and ecosystem, under these scenarios.
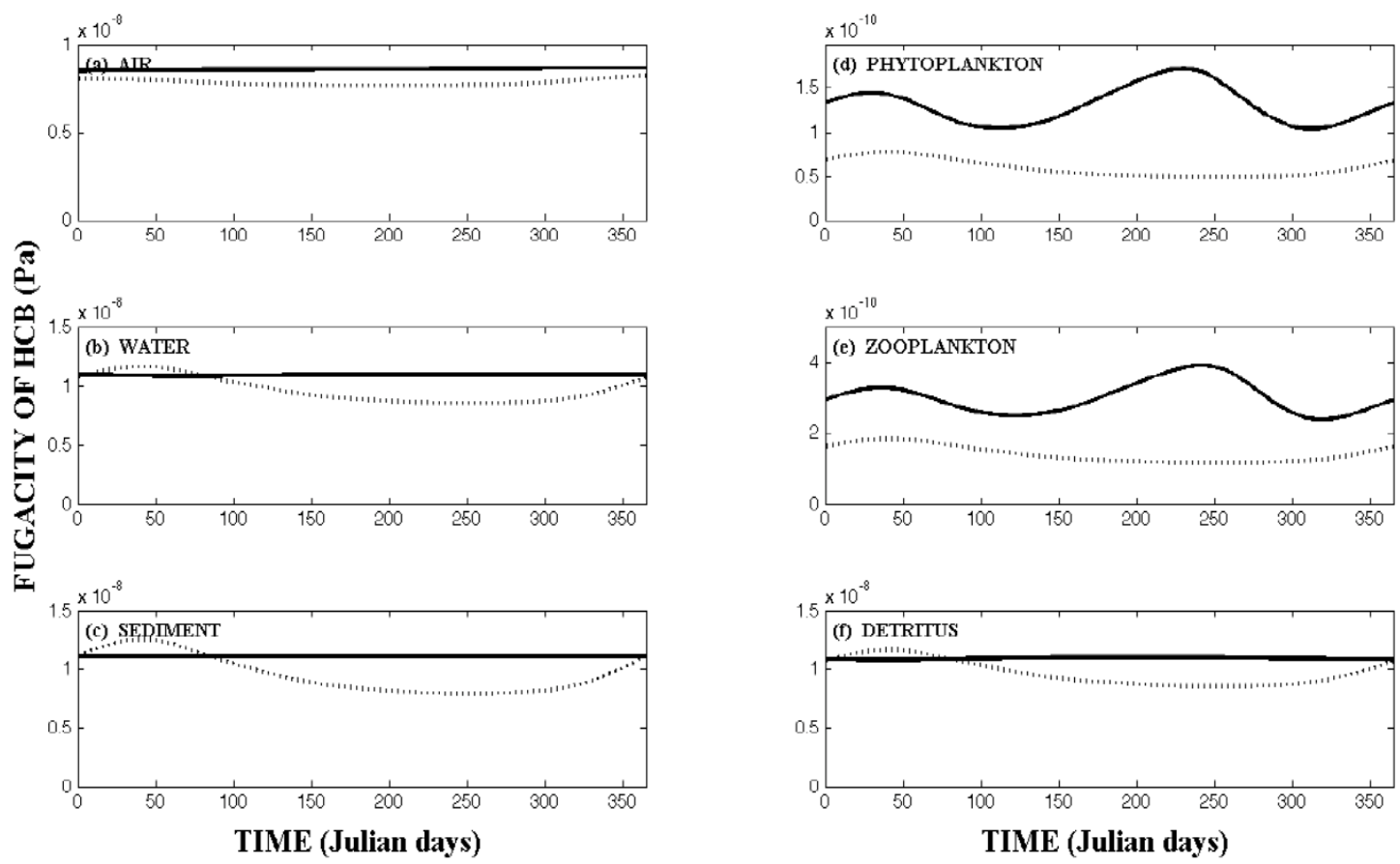

Figure 1. The influences of seasonal ecosystem dynamic (solid line) and temperature (dashed line) variations on fugacity of HCB in the various compartments with time.

The amplitude of the cycling induced by the biology is much less than one percent of the mean fugacity in the air and sediment compartments (Figure 1a,c), 1-2\% in the water and detritus compartments (Figure 1b,f), but $50-60 \%$ in the phytoplankton and zooplankton compartments (Figure 1d,e). The variations in fugacity induced by the temperature variations are $\sim 5 \%$ in air, 30\% in water and detritus, $50 \%$ in sediment, and $20 \%$ 
in phytoplankton and zooplankton. The intra-annual variations in fugacity shown in Figure 1 are therefore confounded by the effect of temperature variations and biomass variations, with temperature being the major driver of seasonal variation of fugacity in the physical compartments and ecosystem dynamics the major driver in the biological compartments. Detritus, although of biological origin, has seasonal variations in fugacity more like the physical phases of sediment and water than the biological phases of the plankton. The very high fugacity capacities of the biological phases (including detritus) ensure that they have the highest concentrations of HCB.

The separate effects of seasonal temperature and ecosystem variations on the mass of HCB in each compartment are shown below in Figure 2.
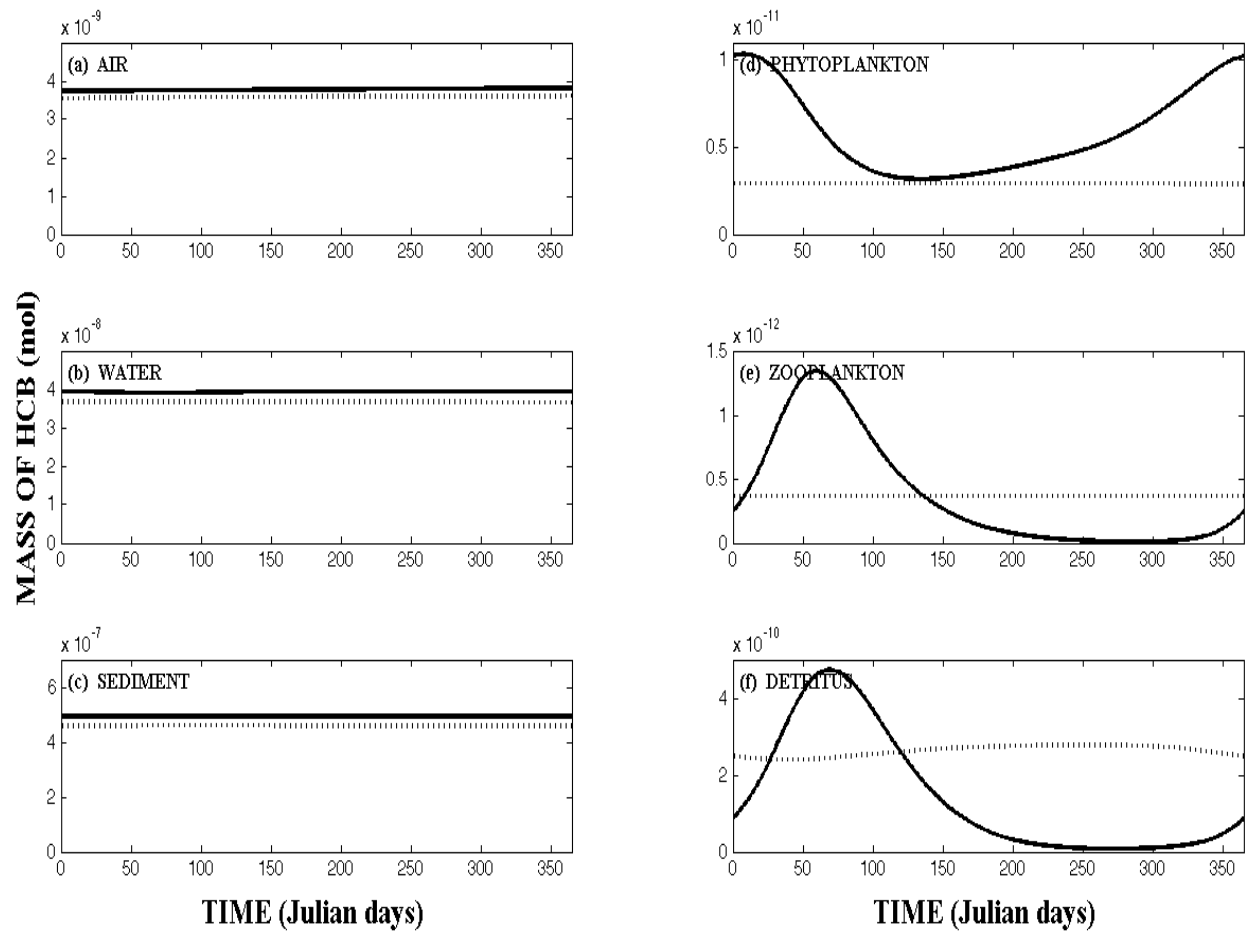

Figure 2. The influences of seasonal ecosystem dynamic (solid line) and temperature (dashed line) variations on the mass of HCB in the various compartments with time.

In comparison with the fugacity (concentration) profiles, the details of the plankton and detritus profiles for HCB mass with time are different, but again, temperature changes do not play a major role compared to ecosystem dynamics. The model predicts that while detritus will have the highest concentration of HCB, because of its very low mass it does not contain as much HCB mass as the physical compartments. However, detritus usually contains more HCB mass than either of the plankton compartments. This result is interesting because detritus is rarely represented as a separate dynamic compartment in models simulating the movement of POPs through ecosystems. Planktonic detritus is often included in a 'suspended solids' compartment that comprises material with different origins and very different properties, and is not dynamically linked to the ecosystem that generates it.

Also of interest is the influence of temperature and ecosystem dynamics of HCB fluxes. The largest diffusive transport of HCB mass in the model occurs between the water and detritus, with maximum flux values at least an order of magnitude larger than the diffusive exchange between the physical compartments, and two to three orders of magnitude larger than the biological compartments. As shown in Figure 3, detritus exchange with sediment is several orders of magnitude lower than its exchange with water because of the tortuous path approximation used for this process (Wania et al., 2006). All movements of mass are with respect to the latter phase shown, therefore positive fluxes indicate a movement of HCB mass into that phase. The sediment-water flux is driven by changes in temperature, whereas the detritus-water flux, which is two orders of magnitude greater than the sediment-water flux, is driven by ecosystem dynamics. 

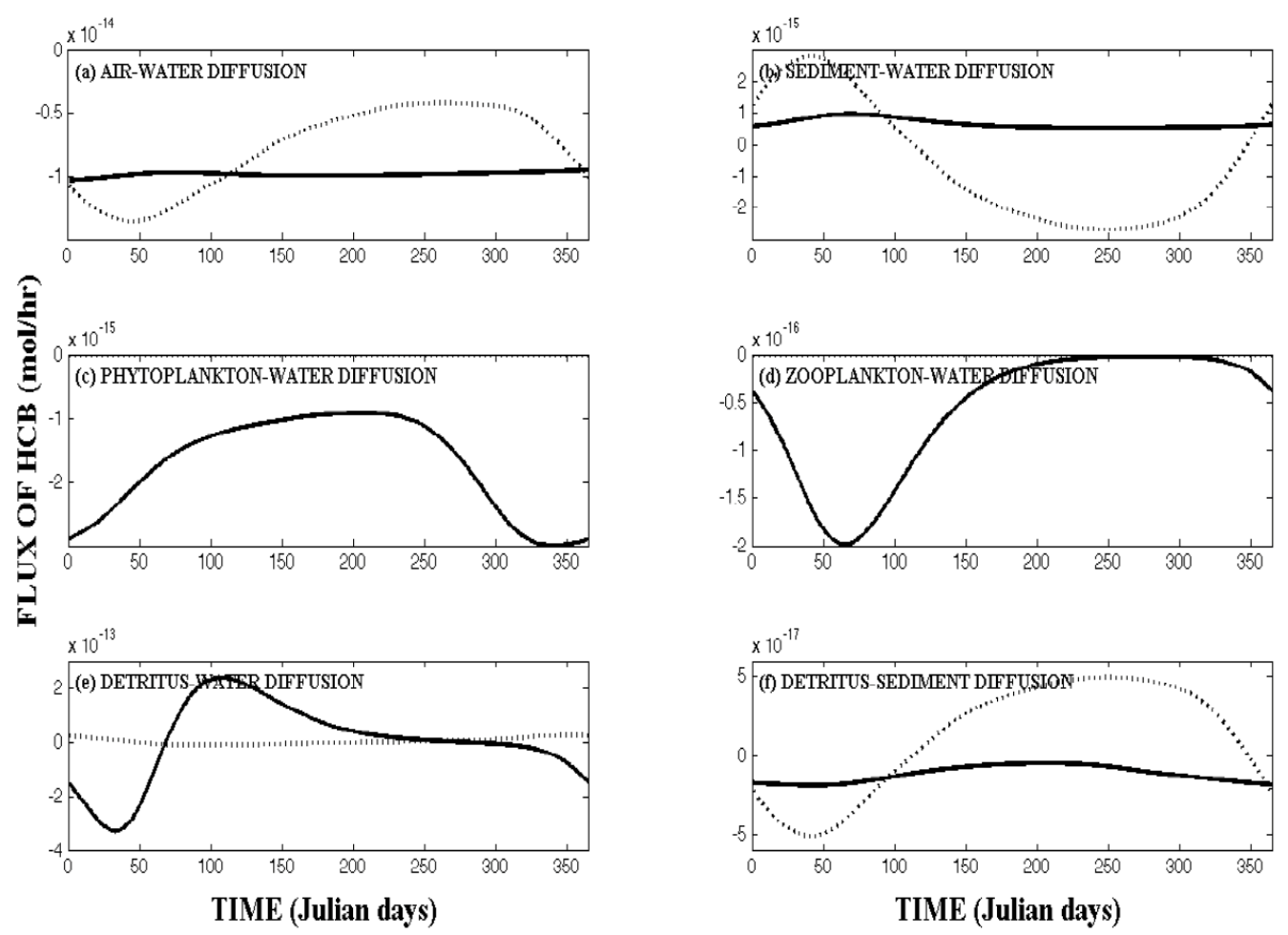

Figure 3. The influences of seasonal ecosystem dynamic (solid line) and temperature (dashed line) variations on inter-compartmental diffusive fluxes of HCB.

The mass fluxes of HCB mass as a result of ecological interactions (Figure 4) are of similar magnitude to those in the physical system. As might be expected, they are driven by ecosystem dynamics.
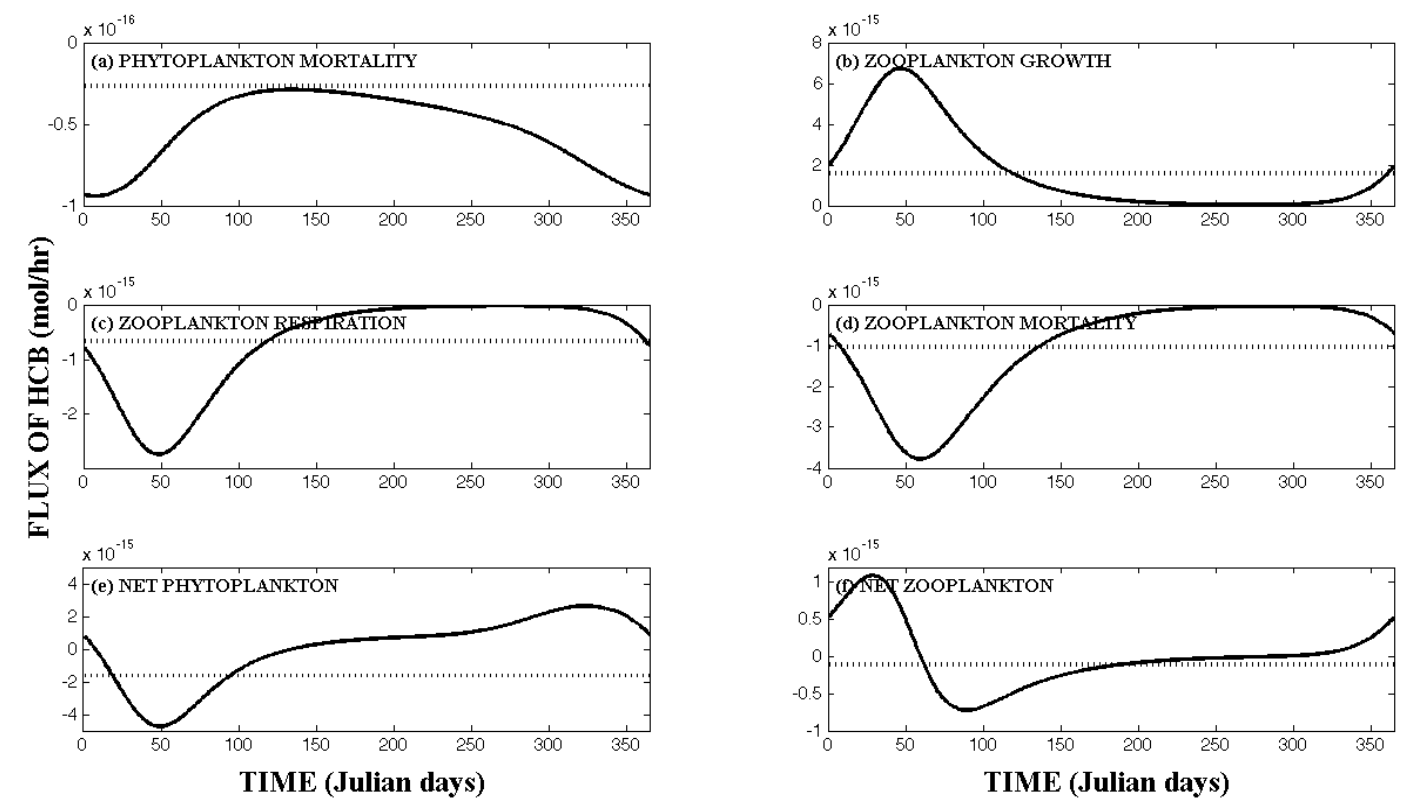

Figure 4. The influences of seasonal ecosystem dynamic (solid line) and temperature (dashed line) variations on fluxes of $\mathrm{HCB}$ as a result of ecosystem processes. 


\section{DISCUSSION AND CONCLUSIONS}

The application of the model to simulate the distribution of HCB in the Antarctic environment has generated some interesting insights into the relative importance of the processes involved. It reveals that food web dynamics can subtly affect the distribution of HCB in the environment, as the seasonal variations observed in the plankton populations are also found in the physical compartments. The model identifies detritus as a compartment with unique properties that couples the physical and biological systems. Detritus is a product of the plankton ecosystem, and so could be expected to share many of the properties of the other ecosystem compartments. However, the fugacity (and hence concentration) of HCB in detritus is mostly driven by temperature, and overwhelms the seasonal variations in detritus driven by variations in the phytoplankton and zooplankton compartments. In these respects, detritus has properties similar to the physical compartments, however, the mass of HCB in detritus has seasonal variations very similar to those of the plankton, and in particular almost identical to zooplankton, confirming its key role in coupling the systems. These results confirm the need for dynamic, coupled POPs modeling in environments such as Antarctica.

\section{REFERENCES}

Bengtson-Nash, S.M. (2011). Persistent organic pollutants in Antarctica: Current and future research priorities. Journal of Environmental Monitoring, 13, 497 - 504.

Bengtson Nash, S.M., Poulsen, A.H., Kawaguchi, S., Vetter, W. and Schlabach, M. (2008). Persistent organohalogen contaminant burdens in Antarctic krill (Euphausia superba) from the eastern Antarctic sector: a baseline study. Science of the Total Environment, 407, 304 - 314.

Corsolini, S., Romeo, T., Ademollo, N., Greco, S. and Focardi, S. (2002). POPs in key species of marine Antarctic ecosystem. Microchemical Journal, 73, 187 - 193.

Cropp, R., Kerr, G., Bengtson-Nash, S. and Hawker D. (2011). A dynamic biophysical fugacity model of the movement of a persistent organic pollutant in Antarctic marine food webs. Environmental Chemistry, 8, $263-280$.

Dachs, J., Eisenreich, S.J., Baker, J.E., Ko, K.-C. and Jeremiason, J.D. (1999) Coupling of phytoplankton uptake and air-water exchange of persistent organic pollutants. Environmental Science and Technology, 33, 3653 - 3660.

De Laender, F., van Ovelen, D., Frantzen, S., Middelburg, J.J. and Soetaert, K. (2010) Seasonal PCB bioaccumulation in an Arctic Marine Ecosystem: A Model Analysis Incorporating Lipid Dynamics, FoodWeb Productivity and Migration. Environmental Science \& Technology, 44, 356 - 61.

Franks, P.J.S. (2002). NPZ Models of Plankton Dynamics: Their construction, coupling to physics, and application. Journal of Oceanography, 58, 379 - 87.

Gabric, A.J., J, Whetton P.H., Cropp R.A. (2001) Dimethylsulphide production in the subantarctic southern ocean under enhanced greenhouse conditions. Tellus, 53(B), 273 - 87.

Hooker, SB, McClain CR. (2000). The Calibration And Validation Of Seawifs Data. Progress in Oceanography, 45, 427-65.

Kelly, B.C., Ikonomou M.G., Blair J.D., Gobas F.A.P.C. (2008). Bioaccumulation behaviour of polybrominated diphenyl ethers (PBDEs) in a Canadian Arctic marine food web. Science of the Total Environment, 401, 60 - 72.

Mackay, D. (2001) Multimedia Environmental Models. The Fugacity Approach, Taylor and Francis, Boca Raton.

Mackay, D., Arnot J.A., Webster E. and Reid L. (2009). Ecotoxicology Modeling: Emerging Topics in Ecotoxicology: Principles, approaches and Perspectives 2, pp. 355. Springer, Dordrecht.

Reynolds, C. (2006). Ecology of Phytoplankton, University of Cambridge, Cambridge.

Shen, L. and Wania F. (2005). Compilation, Evaluation, and Selection of Physical-Chemical Property Data for Organochlorine Pesticides. Journal of Chemical \& Engineering Data, 50, 742 - 68.

Stearns, C.R., Keller L.M., Weidner G.A. and Sievers M. (1993). Antarctic Meteorology and Climatology: Studies based on automatic weather stations pp. 1. American Geophysical Union, Washington, DC.

Wania F. and Mackay, D. (1995). A global distribution model for persistent organic chemcials. The Science of the Total Environment, 160, 211-32.

Wania, F. and Mackay, D. (1999). The evolution of mass balance models of persistent organic pollutant fate in the environment. Environmental Pollution, 100, $223-40$.

Wania, F., Breivik K., Persson N.J., McLachlan M.S. (2006). CoZMo-POP2 A fugacity-based dynamic multi-compartmental mass balance model of the fate of persistent organic pollutants. Environmental Modelling and Software, 21, 868-84.

Weber, K. and Goerke H. (2003) Persistent organic pollutants (POPs) in antarctic fish: levels, patterns, changes. Chemosphere, 53, $667-78$. 